\title{
Substrate temperature influence on the properties of GaN thin films grown by hollow-cathode plasma-assisted atomic layer deposition
}

\author{
Mustafa Alevlia) and Neşe Gungor \\ Department of Physics, Faculty of Arts and Sciences, Marmara University, Goztepe, 34722 Istanbul, Turkey \\ Ali Haider, Seda Kizir, Shahid A. Leghari, and Necmi Biyikli ${ }^{\text {b) }}$ \\ Institute of Materials Science and Nanotechnology, Bilkent University, Bilkent, 06800 Ankara, Turkey \\ and National Nanotechnology Research Center (UNAM), Bilkent University, Bilkent, 06800 Ankara, Turkey
}

(Received 27 August 2015; accepted 10 November 2015; published 20 November 2015)

\begin{abstract}
Gallium nitride films were grown by hollow cathode plasma-assisted atomic layer deposition using triethylgallium and $\mathrm{N}_{2} / \mathrm{H}_{2}$ plasma. An optimized recipe for $\mathrm{GaN}$ film was developed, and the effect of substrate temperature was studied in both self-limiting growth window and thermal decomposition-limited growth region. With increased substrate temperature, film crystallinity improved, and the optical band edge decreased from 3.60 to $3.52 \mathrm{eV}$. The refractive index and reflectivity in Reststrahlen band increased with the substrate temperature. Compressive strain is observed for both samples, and the surface roughness is observed to increase with the substrate temperature. Despite these temperature dependent material properties, the chemical composition, $E_{1}(\mathrm{TO})$, phonon position, and crystalline phases present in the GaN film were relatively independent from growth temperature. (C) 2015 American Vacuum Society.
\end{abstract}

[http://dx.doi.org/10.1116/1.4936230]

\section{INTRODUCTION}

$\mathrm{GaN}$ is among the most notable III-V compounds with its excellent properties, such as wide direct bandgap, high thermal stability, and high electron velocities, which make it an attractive candidate for short wavelength optoelectronics and high-power and high-frequency electronics. ${ }^{1,2}$ Although high-quality GaN films grown by metal-organic chemical vapor deposition ${ }^{3,4}$ and molecular beam epitaxy ${ }^{5}$ at high growth temperatures $\left(>800^{\circ} \mathrm{C}\right)$, which is necessary to increase the cracking efficiency of ammonia $\left(\mathrm{NH}_{3}\right)$ and mobility of precursor species at the substrate surface, ${ }^{6}$ high growth temperatures limit the integration of GaN-based optoelectronic devices with Si CMOS technology as well as not being suitable for deposition of $\mathrm{GaN}$ on flexible polymeric substrates. Moreover, the integration of narrow band gap (In-rich) with wide band gap (Ga-rich) III-nitride layers is not possible due to the low vapor pressure and disassociation temperature of $\mathrm{InN}^{7}{ }^{7}$ This restricts the development of highly efficient III-nitride multijunction solar cells and spectral tunable light sources. In order to overcome the limitations due to high substrate temperatures, most prominent alternative approaches for low-temperature growth of $\mathrm{GaN}$ include physical vapor deposition techniques (sputtering and pulsed laser deposition) as well as a chemical vapor deposition technique: plasma-assisted atomic layer deposition (PAALD).$^{8-10}$ When compared with PVD techniques, PA-ALD stands out in terms of large area uniformity, submonolayer precision thickness control, and ultimate conformality. ${ }^{11}$

There are only a few recent reports on the growth of GaN films using atomic layer deposition at low temperatures $\left(<300^{\circ} \mathrm{C}\right)$ with improved crystalline quality and electrical

\footnotetext{
a)Electronic mail: mustafaalevli@marmara.edu.tr

b)Electronic mail: biyikli@unam.bilkent.edu.tr
}

properties. $^{6,12,13}$ In these reports, PA-ALD of GaN using trimethylgallium, ${ }^{12}$ triethylgallium (TEG), ${ }^{6,13}$ and $\mathrm{N}_{2} / \mathrm{H}_{2}$ plasma as the precursors were reported at relatively low substrate temperatures. However, yet there is no report on the effect of the substrate temperature on the structural and optical properties of GaN films grown by using hollow-cathode plasma-assisted atomic layer deposition (HCPA-ALD). An optimized ALD process window was developed in the selflimiting growth regime $\left(185-385^{\circ} \mathrm{C}\right),{ }^{10}$ in which saturation for both precursors are satisfied along with constant growth rate per cycle. Details of the optimization results will be published elsewhere. Following the growth of GaN film within the ALD window, GaN films were deposited at higher substrate temperatures within the decomposition limited region in which CVD type of deposition takes place and growth rate increased with substrate temperature. ${ }^{14}$

In this work, we have reported the effect of the substrate temperatures $\left(200\right.$ and $450^{\circ} \mathrm{C}$ ) on the structural and optical properties of HCPA-ALD grown GaN films on Si (100) and quartz substrates. A comparative analysis of structural, compositional, refractive index, optical phonon modes, and optical band edge is demonstrated.

\section{EXPERIMENT}

GaN samples were grown using a modified Fiji F200-LL ALD reactor (Ultratech/CambridgeNanotech, Inc.) in which quartz-based inductively coupled RF-plasma source was replaced with a stainless steel hollow-cathode plasma source (Meaglow Inc.). GaN films were grown with identical parameters except substrate temperatures of 200 and $450{ }^{\circ} \mathrm{C}$. TEG was kept at room temperature and used as the gallium precursor. The TEG exposure doses were adjusted in order to obtain the self-limiting growth scheme which is an evidence of ideal ALD growth mechanism. Three-hundred watt 
remote rf-plasma assisted decomposition of nitrogen/hydrogen $\left(\mathrm{N}_{2} / \mathrm{H}_{2}\right)$ mixture was utilized as a nitrogen precursor. In addition, the total numbers of deposition cycle were adjusted to achieve similar film thickness (1750 cycles for sample grown at $200^{\circ} \mathrm{C}$ and 900 cycles for sample grown at $450^{\circ} \mathrm{C}$ ). Based on the optimization studies, each ALD cycle consisted of four steps: $0.5 \mathrm{~s}$ TEG, $10 \mathrm{~s}$ Ar purge, $40 \mathrm{~s} 50+$ $50 \mathrm{sccm} \mathrm{N} / \mathrm{H}_{2}$ plasma, and $10 \mathrm{~s}$ Ar purge.

Structural properties of the GaN films grown on $\mathrm{Si}(100)$ substrates were studied by using a PanAnalytical X'Pert PRO MRD system using $\mathrm{Cu} K \alpha$ radiation in $\Theta-2 \Theta$ mode. Grazing-incidence x-ray diffraction (GIXRD) has been performed in the range of $25^{\circ}-75^{\circ}$. Incidence angle $(\omega)$ was $0.3^{\circ}$ for GIXRD measurements. GIXRD patterns were obtained by performing scans with a step size of $0.1^{\circ}$. Peak positions were obtained by fitting the GIXRD data using PANALYTICAL X'PERT DATA VIEWER software. X-ray photoelectron spectroscopy (XPS) (Thermo Scientific) measurements were carried out using $\mathrm{Al} \mathrm{K} \alpha$ radiation. The pass energy, step size, and spot size were $30 \mathrm{eV}, 0.1 \mathrm{eV}$, and $400 \mu \mathrm{m}$, respectively. Peak deconvolution was performed using the AVANTAGE software, without applying any restrictions to spectral location and full width at half maximum values. The surface morphology was investigated by using atomic force microscopy (AFM, Park systems Corp., XE-100). The film thicknesses and optical constants were measured by spectroscopic ellipsometer (SE) (V-VASE, J.A. Woollam Co., Inc.) in the wavelength range of $300-1000 \mathrm{~nm}$ at various incidence angles. In order to evaluate the film thickness and optical band gap, the UV-visible room temperature optical transmission measurements were carried out in the 215-1000 nm wavelength range on GaN films deposited on quartz substrates with a resolution of $1 \mathrm{~nm}$. Fourier transform infrared reflectance (FTIR) spectra were taken at room temperature and at normal incidence of IR radiation in the range of $450-700 \mathrm{~cm}^{-1}(22.22-14.28 \mu \mathrm{m})$ with a spectral resolution of $2 \mathrm{~cm}^{-1}$ by using a FTIR spectrometer (Bruker Vertex 70) with a mirror optics microscope (hyperion microscope) and a liquid nitrogen cooled MCT detector. All spectra were normalized to the reflection of a gold mirror.

\section{RESULTS AND DISCUSSION}

\section{A. Structural, compositional, and surface analyses}

Grazing incidence $\mathrm{x}$-ray diffraction was used to analyze the structure of HCPA-ALD grown GaN films. Increasing the substrate temperature affected neither peak positions nor the diffraction planes. Figure 1 shows the crystalline phases of the $\sim 60 \mathrm{~nm}$ thick GaN films grown at different temperatures. The patterns include three main Bragg peaks corresponding to (002), (103) reflections, and a peak that encloses the (200), (112), and (201) reflections of the hexagonal GaN phase. The sharp diffraction peaks showed no other phase or impurity related diffraction peak, which validates the relatively high purity and crystalline structure of both GaN films. Similar XRD patterns were obtained for GaN films deposited at different substrate temperatures. It can be seen that the GIXRD peak intensities increased with substrate temperature,

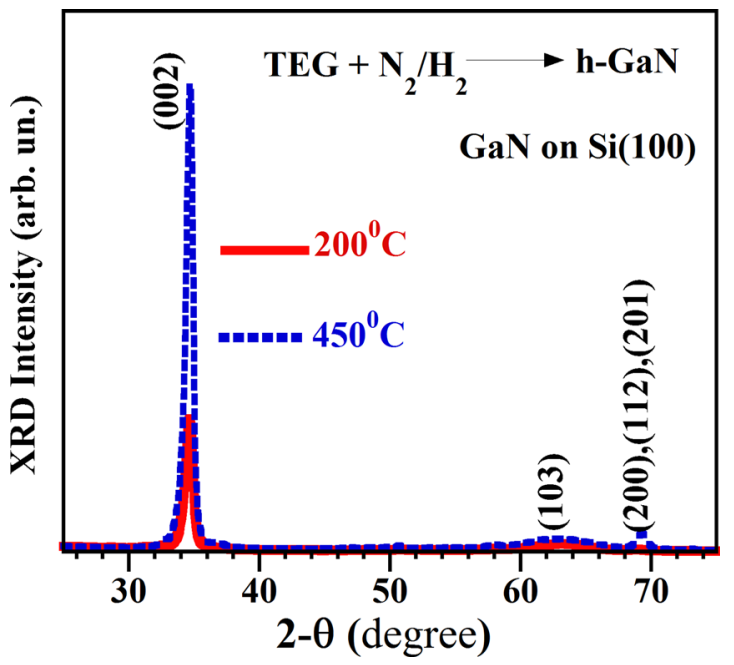

FIG. 1. (Color online) GIXRD patterns of GaN films as a function of $2 \Theta$ deposited at 200 and $450^{\circ} \mathrm{C}$ on $\mathrm{Si}(100)$ with the growth parameters obtained in the ALD window.

indicating a slight improvement in crystalline quality of $\mathrm{GaN}$, which might be due to faster reaction and mass transport kinetics. ${ }^{14}$ The lattice parameters $(a$ and $c$ ) for GaN films were roughly calculated from the peak positions using the famous Bragg's law. The calculated lattice constants in $c$-axis were $5.176 \pm 0.00003$ and $5.167 \pm 0.00003 \AA$, and the calculated lattice in $a$-axis were $3.184 \pm 0.00003$ and $3.185 \pm 0.00003 \AA$ for the $\mathrm{GaN}$ samples grown at 200 and $450{ }^{\circ} \mathrm{C}$, respectively. These values are in good agreement with unstrained $\mathrm{GaN}\left(a_{0}=3.189 \AA, c_{0}=5.185 \AA\right){ }^{15}$ It should be noted that the lattice constant $a$ of GaN films did not change with the substrate temperature while the lattice constant $c$ decreased. The disharmony in lattice constants with the increasing substrate temperature was correlated with the compressive strain in the films. The elastic strains parallel $\left(\varepsilon_{/ /}\right)$ to the $c$-axis and vertical $\left(\varepsilon_{\perp}\right)$ to the $c$-axis were calculated by using the following expressions: $\varepsilon_{/ /}=\left(a_{\mathrm{epi}}-a_{0}\right) / a_{0}$ and $\varepsilon_{\perp}=\left(c_{\text {epi }}-c_{0}\right) / c_{0} \cdot{ }^{15} c_{\text {epi }}, a_{\text {epi }}, c_{0}$, and $a_{0}$ are the lattice constants for epilayer and bulk material, respectively. The calculated parallel strain were $-0.15 \pm 0.006$ and $-0.12 \pm 0.006$, and the vertical strain were $-0.17 \pm 0.006$ and $-0.34 \pm 0.006$ for the films grown at 200 and $450{ }^{\circ} \mathrm{C}$, respectively. The calculated values of in-plane $\left(\varepsilon_{/ /}\right)$strain show the existence of very small compressive strain for both GaN films. On the other hand, the calculated negative values of out plane $\left(\varepsilon_{\perp}\right)$ strain show the existence of compressive strain along the $c$ axis for both $\mathrm{GaN}$ films with increasing substrate temperature. The calculated values of $\varepsilon_{\perp}$ and $\varepsilon_{/ /}$indicate the existence of compressive strain in all samples.

Furthermore, the average crystallite sizes of the GaN films were calculated from the (002) reflections using Scherer equation and found to be $12.98 \pm 0.0003 \mathrm{~nm}\left(200^{\circ} \mathrm{C}\right)$ and $14.26 \pm 0.0003 \mathrm{~nm}\left(450^{\circ} \mathrm{C}\right)$. It can be concluded that the crystallite size increased with substrate temperature, which confirms the improved GaN crystalline quality.

We explored the influence of substrate temperatures on the compositional properties of GaN films by using the aforementioned ALD growth conditions. Figure 2 shows the 


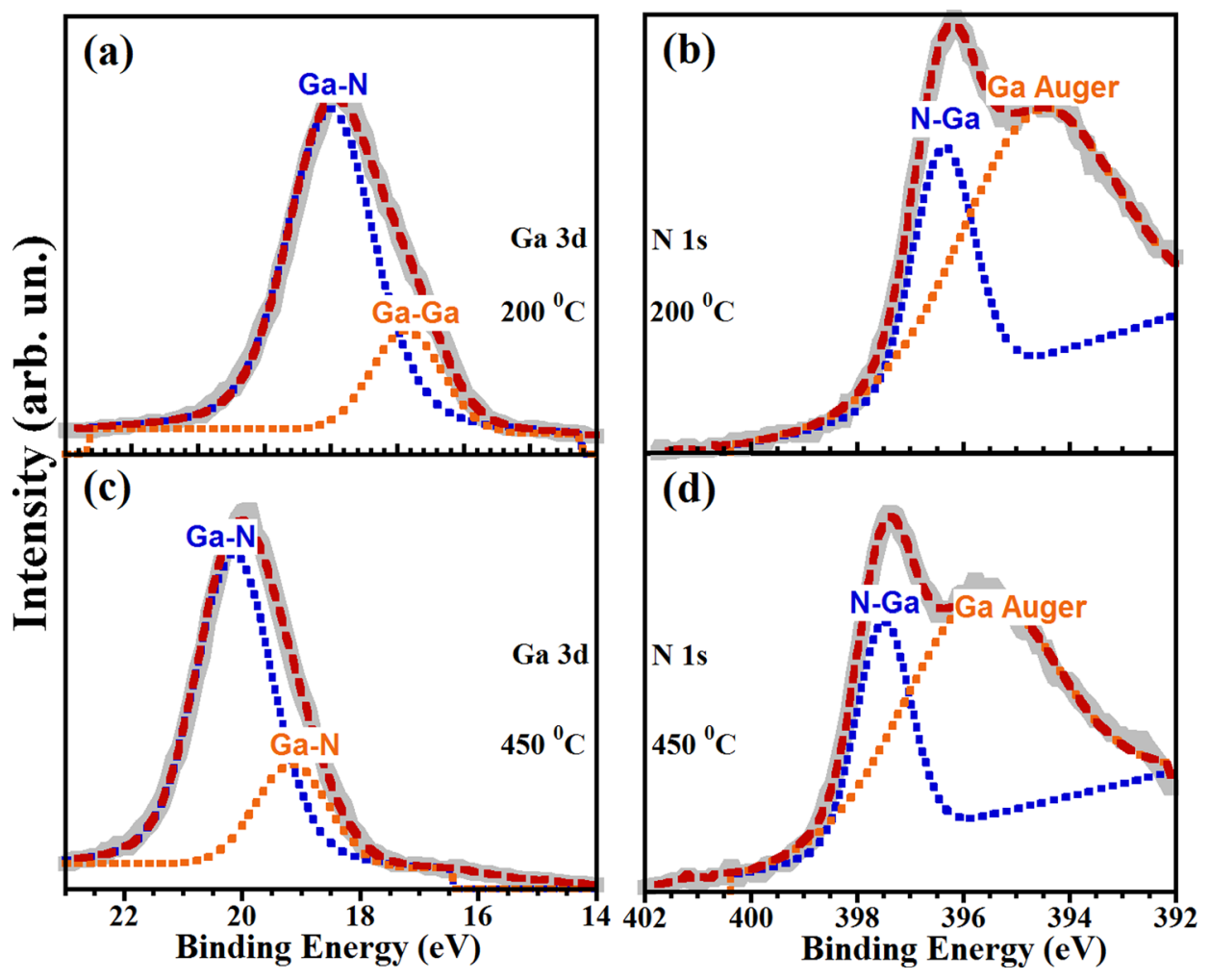

FIG. 2. (Color online) High resolution XPS spectra of (a) Ga $3 d$, and (b) $\mathrm{N} 1 s$ for $\mathrm{GaN}$ film grown at $200^{\circ} \mathrm{C}$ and (c) $\mathrm{Ga} 3 d$, and (d) $\mathrm{N} 1 s$ for $\mathrm{GaN}$ film grown at $450{ }^{\circ} \mathrm{C}$.

evolution of Ga $3 d$ [Figs. 2(a) and 2(c)] and N $1 s$ [Figs. 2(b) and 2(d)] high resolution XPS (HR-XPS) spectra for different substrate temperatures $\left\{200^{\circ} \mathrm{C}\right.$ [Figs. 2(a) and 2(b)] and $450^{\circ} \mathrm{C}$ [Figs. 2(c) and 2(d)] \}. The HR-XPS spectrum of Ga $3 d$ [Fig. 2(a)] for GaN film grown at $200^{\circ} \mathrm{C}$ can be decomposed into two components centered at 19.0 and $17.85 \mathrm{eV}$ and assigned to $\mathrm{Ga}-\mathrm{N}$ and $\mathrm{Ga}-\mathrm{Ga}$ bonding states, respectively. ${ }^{12,16} \mathrm{~N} 1 s$ spectrum [Fig. 2(b)] for GaN film grown at $200{ }^{\circ} \mathrm{C}$ can also be similarly decomposed into two components at 396.37 and $394.57 \mathrm{eV}$, which is assigned to the $\mathrm{N}-\mathrm{Ga}$ bond and Auger Ga peaks. ${ }^{12}$ Although the spectral locations and intensities of $\mathrm{Ga} 3 d$ and $\mathrm{N} 1 s$ peaks were found to be slightly different for $\mathrm{GaN}$ samples grown at $450^{\circ} \mathrm{C}$, the atomic composition of GaN film was hardly changed. The HR-XPS spectrum of Ga $3 d$ [Fig. 2(c)] for GaN film grown at $450^{\circ} \mathrm{C}$ was fitted by two subpeaks located at 20.10 and $19.15 \mathrm{eV}$, corresponding to Ga-N bonds. ${ }^{12}$ The $\mathrm{N} 1 s$ spectrum [Fig. 2(d)] obtained for GaN grown at $450^{\circ} \mathrm{C}$ was fitted similarly using two subpeaks located at 397.49 and $395.67 \mathrm{eV}$, corresponding to $\mathrm{N}-\mathrm{Ga}$ and Auger Ga bonds, respectively. ${ }^{12,16}$ As the deposition temperature increases, the $\mathrm{Ga} 3 d$ and $\mathrm{N} 1 s$ binding energies (BEs) shifted from lower $\mathrm{BE}$ to the higher $\mathrm{BE}$. The shift in the Ga $3 d$ and $\mathrm{N} 1 s$ core levels was observed to be $1 \mathrm{eV}$. This shift might be attributed to a change in band bending, which is a result of oxygen reduction in the $\mathrm{GaN}$ structure. This explanation agrees well with the atomic percentage of oxygen obtained from the XPS analysis. The atomic percentage of oxygen is 3.24 and 1.71 at. \% for $\mathrm{GaN}$ films grown at 200 and $450{ }^{\circ} \mathrm{C}$, respectively. Nevertheless, no carbon signals were observed by the XPS measurement, indicating that ligands consisting of ethyl groups in TEG were almost completely removed during the exposure of the $\mathrm{N}_{2} / \mathrm{H}_{2}$ plasma.

To further investigate the difference between the GaN films grown on ALD window and decomposition limited region, the surface morphology was characterized by AFM. The surface roughness and grain size of $\mathrm{GaN}$ films were obtained from AFM images [Figs. 3(a) and 3(b)]. Root mean square surface roughness values measured from a $1 \times 1 \mu \mathrm{m}^{2}$ scan area of GaN samples grown at 200 and $450{ }^{\circ} \mathrm{C}$ were recorded as 0.36 and $0.98 \mathrm{~nm}$, respectively. The surface of the film grown in self-limiting growth region is very smooth and mostly two-dimensional with several bumps compared to the film grown at $450{ }^{\circ} \mathrm{C}$ with hillocks larger in diameter. As a result, $\mathrm{GaN}$ film grown at higher temperatures exhibited substantially higher surface roughness. The mean grain sizes were obtained from the analysis of AFM images as 5.6 and $5.9 \mathrm{~nm}$ for $\mathrm{GaN}$ films grown at 200 and $450^{\circ} \mathrm{C}$, respectively. Although there is no significant difference in the AFMmeasured grain size, the surface roughness becomes rougher for GaN films grown out of the ALD temperature window due to the effect of the growth temperature in terms of growth speed along different crystallographic directions. ${ }^{13}$

\section{B. Optical properties}

The refractive index $(n)$, the extinction coefficient $(k)$, and optical band edge values $\left(E_{g}\right)$ were determined from spectroscopic ellipsometry measurements and are shown in Fig. 4. In order to model the spectroscopic ellipsometry spectra, we followed two steps: Cauchy dispersion function 

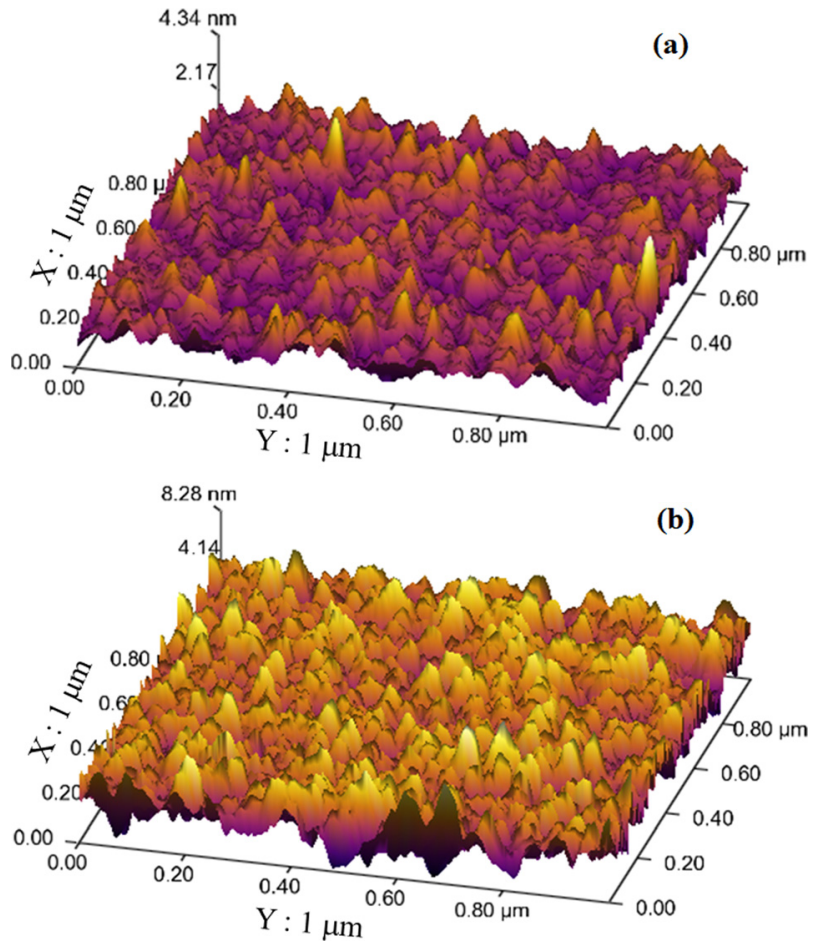

FIG. 3. (Color online) AFM images of $\mathrm{GaN}$ films grown at (a) $200^{\circ} \mathrm{C}$ (b) $450{ }^{\circ} \mathrm{C}$.

was used for transparent wavelength region as the first step. In the following step, by using the optical constants obtained from the first step, Tauc-Lorentz model was used for the absorbing wavelength region of GaN films. The measured ellipsometry data were fitted by assuming two layer structures with a bulklike GaN layer on the Si substrate and a top surface roughness layer for the film deposited at $450{ }^{\circ} \mathrm{C}$. The rough layer is modeled as a mixture of bulk material and void layer. The fitting results provide thickness values for $\mathrm{GaN}$ film deposited at $450{ }^{\circ} \mathrm{C}$ as the following: $54.1 \mathrm{~nm}$ for bulk layer and $14.8 \mathrm{~nm}$ for rough $\mathrm{GaN}$ layer. On the other hand, the measured ellipsometry data for the GaN film deposited at $200{ }^{\circ} \mathrm{C}$ was fitted successfully by assuming merely single $\mathrm{GaN}$ bulk layer and the thickness of $\mathrm{GaN}$ layer is found to be $49.0 \mathrm{~nm}$. There is a good correlation between the fractional thickness of the GaN film with the top rough layer deposited at $450{ }^{\circ} \mathrm{C}$ and the measured surface roughness, which is measured as $0.98 \mathrm{~nm}$. This justifies the two-layer model used for the analysis of ellipsometry data. The formation of the rough layer with the increasing substrate temperature manifests the increase in surface roughness of the GaN films. The value of refractive index at $632 \mathrm{~nm}$ is 2.38 and 2.18 for the $\mathrm{GaN}$ films deposited at 450 and $200^{\circ} \mathrm{C}$, respectively. The evaluation of the refractive index of $\mathrm{GaN}$ is plotted in Fig. 4(a), showing a strong substrate temperature dependence of $n(\lambda)$. This temperature dependence of the refractive index can be due to the variation in crystalline orientation or near-random orientation of crystallites, surface morphology, and improved crystallinity. ${ }^{17,18}$ Refractive indices obtained for both GaN films are consistent with the refractive index value of a typical polycrystalline GaN film. ${ }^{17}$ The absorption coefficient, $\alpha(\lambda)=4 \pi k(\lambda) / \lambda$, was calculated from
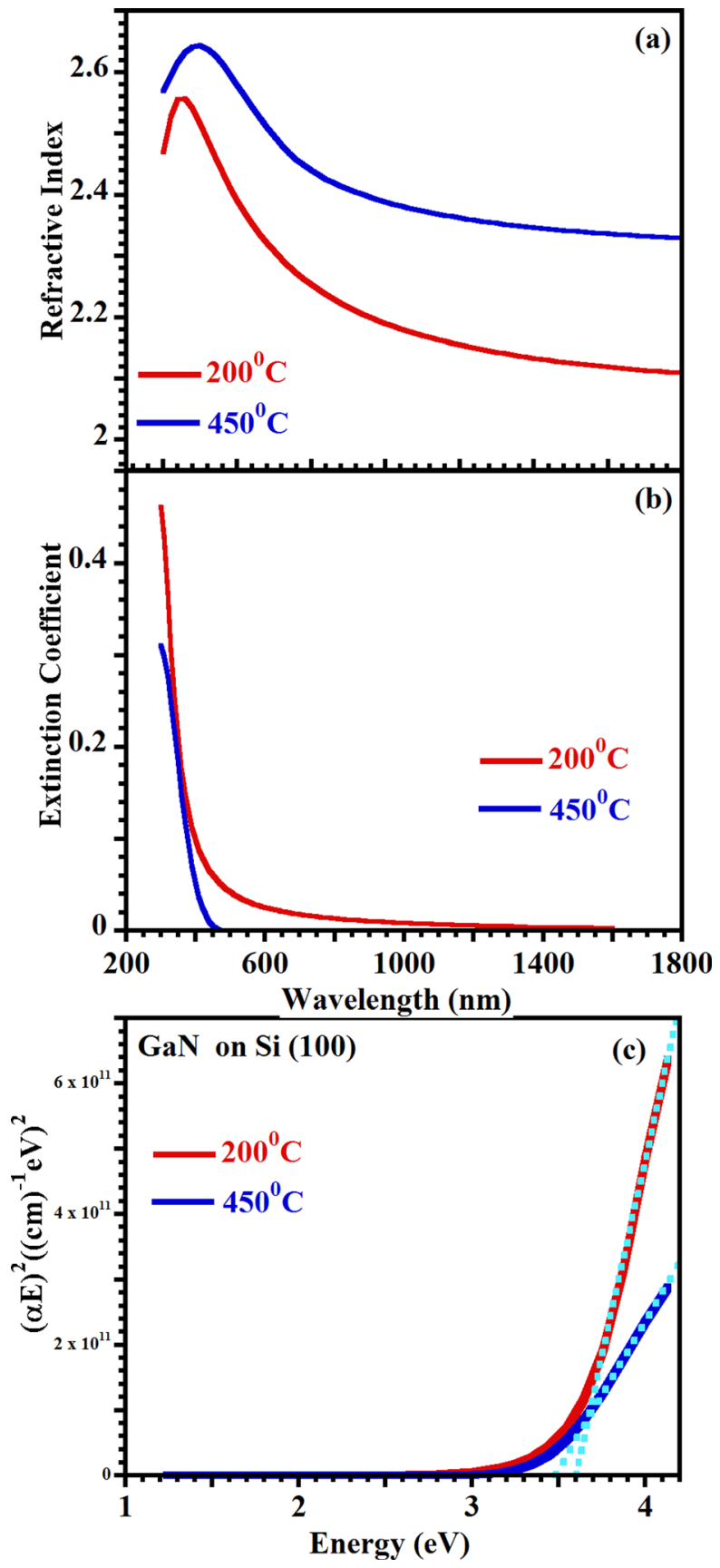

FIG. 4. (Color online) Spectral dispersion of (a) refractive index and (b) extinction coefficient of GaN films deposited at substrate temperatures of 200 and $450^{\circ} \mathrm{C}$. (c) Optical band edge obtained from the spectroscopic ellipsometry data.

the $k(\lambda)$ values determined from ellipsometry. The approximate optical band gap value was obtained via extrapolation of the linear part of the absorption spectrum to $(\alpha E)^{2}=0$. It is found that the band gap decreases with the substrate temperature from 3.60 to $3.52 \mathrm{eV}$.

The optical transmission measurements of GaN films grown on quartz substrates were carried out within the 215-1000 nm wavelength range. Figure 5 shows the optical transmission spectra of GaN layers together with the calculated spectra. The optical transmission spectra were fitted by applying a model dielectric function $(\mathrm{MDF})^{7}$ with additional 


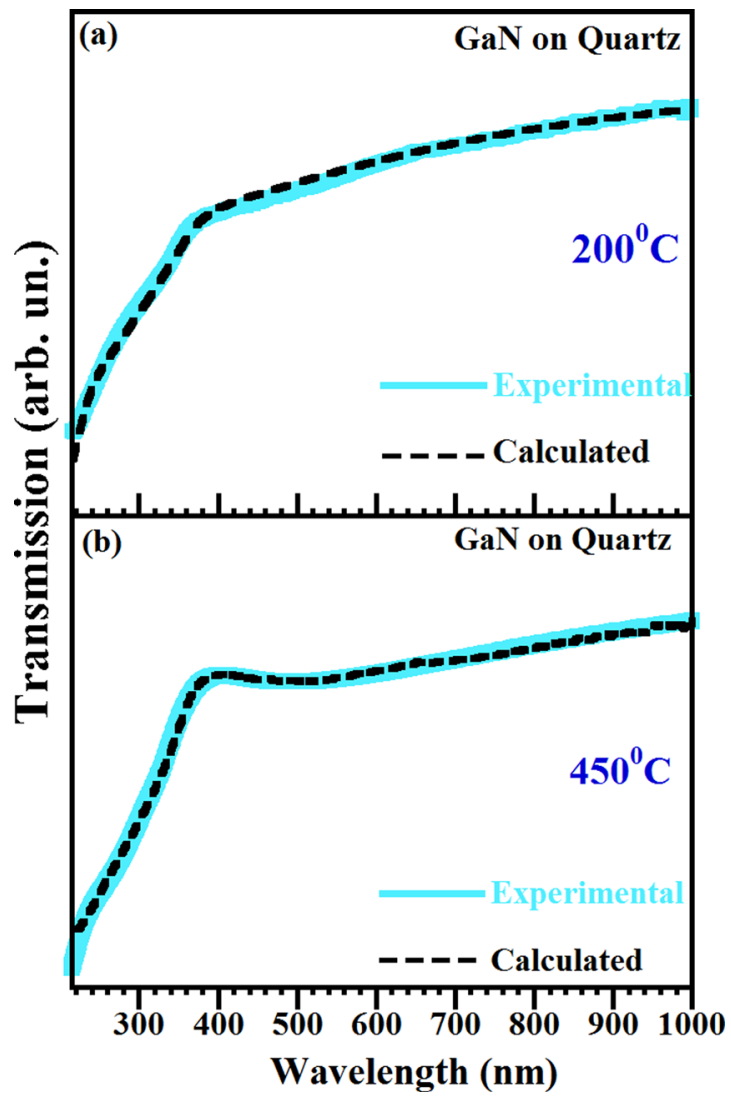

FIG. 5. (Color online) Experimental and best fit calculated transmission spectra of GaN films grown on quartz substrates at different substrate temperatures.

oscillators. This approach provided a good fit of experimental transmission spectra, where the MDF is defined as

$$
\varepsilon_{\mathrm{GaN}}^{\prime}(\omega)=\varepsilon_{\mathrm{GaN}}+\sum_{n=1}^{m} \frac{S_{a b}}{\left[E_{a b}^{2}-(h \omega)^{2}\right]-i h \omega \Gamma_{a b}} .
$$

Here, $m$ is the number of additional absorption centers, $\varepsilon_{\mathrm{GaN}}$ is the dielectric function of $\mathrm{GaN},{ }^{19} S_{a b}$ is the oscillator strength, $E_{a b}$ is the energetic position of the oscillator, and $\Gamma_{a b}$ is the damping of the Lorentzian. From Fig. 5, it is seen that there is a good agreement between the simulated and experimental data (solid line). The optical band gap values were found to be 3.55 and $3.50 \mathrm{eV}$ for films grown at 200 and $450{ }^{\circ} \mathrm{C}$, respectively. The two numbers are both higher than the bulk value of $3.4 \mathrm{eV}$ and consistent with the optical band gap values obtained from SE analysis. The difference in the optical band gap may be attributed crystal disorder, dislocations, and grain boundary misorientations. ${ }^{17,18}$

Figure 6 shows the room temperature experimental (solid line) and calculated infrared reflectance spectrum for $\mathrm{GaN}$ films grown on $\mathrm{Si}(100)$ at $200^{\circ} \mathrm{C}$ [Fig. 6(a)] and $450^{\circ} \mathrm{C}$ [Fig. 6(b)]. Since no additional information was obtained in the high-frequency range above $700 \mathrm{~cm}^{-1}$, we will focus on the Reststrahlen band between 500 and $700 \mathrm{~cm}^{-1}$, which is centered about $570 \mathrm{~cm}^{-1}$. The theoretical analysis of the FTIR reflectance from this $\mathrm{GaN} / \mathrm{Si}$ sample can be applied to obtain further information on the optical phonon. Theoretical IR spectra were calculated using the conventional transfer matrix method where we used $n=3.42$ for the refractive index of $\mathrm{Si}$.

The dielectric functional GaN layer is modeled using Eq. (2) assuming two contributions, two Lorentz oscillators for lattice contribution ${ }^{20}$

$$
\begin{aligned}
\varepsilon(\omega)= & \varepsilon_{\infty}\left(\prod_{i=1}^{2} \frac{\omega^{2}+i \gamma_{\mathrm{LO}, i} \omega-\omega_{\mathrm{LO}, i}^{2}}{\omega^{2}+i \gamma_{\mathrm{TO}, i} \omega-\omega_{\mathrm{TO}, i}^{2}}\right. \\
& \left.+\sum_{j=1}^{5}\left(\frac{S_{J} \omega_{\mathrm{TO}, j}^{2}}{\omega_{\mathrm{TO}_{j}, j}^{2}-\omega^{2}-i \omega \Gamma_{j}}\right)\right) .
\end{aligned}
$$

Here, $\varepsilon_{\infty}, \omega_{\mathrm{LO}, i j}, \gamma_{\mathrm{LO}, i j}, \omega_{\mathrm{TO}, i j}$, and $\gamma_{\mathrm{TO}, i j}$ are, in order, high frequency dielectric frequency, the frequency and the broadening value of the $i$ th LO and TO phonon, $S_{i j}$ denotes the strength, and $\Gamma_{i j}$ the damping constant of the $i$ th lattice mode. The free-carrier effects are neglected since the $\mathrm{GaN}$ layers are undoped. The experimental IR spectra for both GaN samples agree well with the calculated Reststrahlen band for a single crystalline wurtzite GaN film grown on $\mathrm{Si}(100)$. There is no significant discrepancy between the spectra of $\mathrm{GaN}$ samples. The reflectance intensity of Reststrahlen band increases with the deposition temperature, which is a result of improved crystallinity. Two main peaks $(A, B)$ and one dip $(C)$ in the IR spectra are the main features observed in the literature for epitaxial GaN films grown on

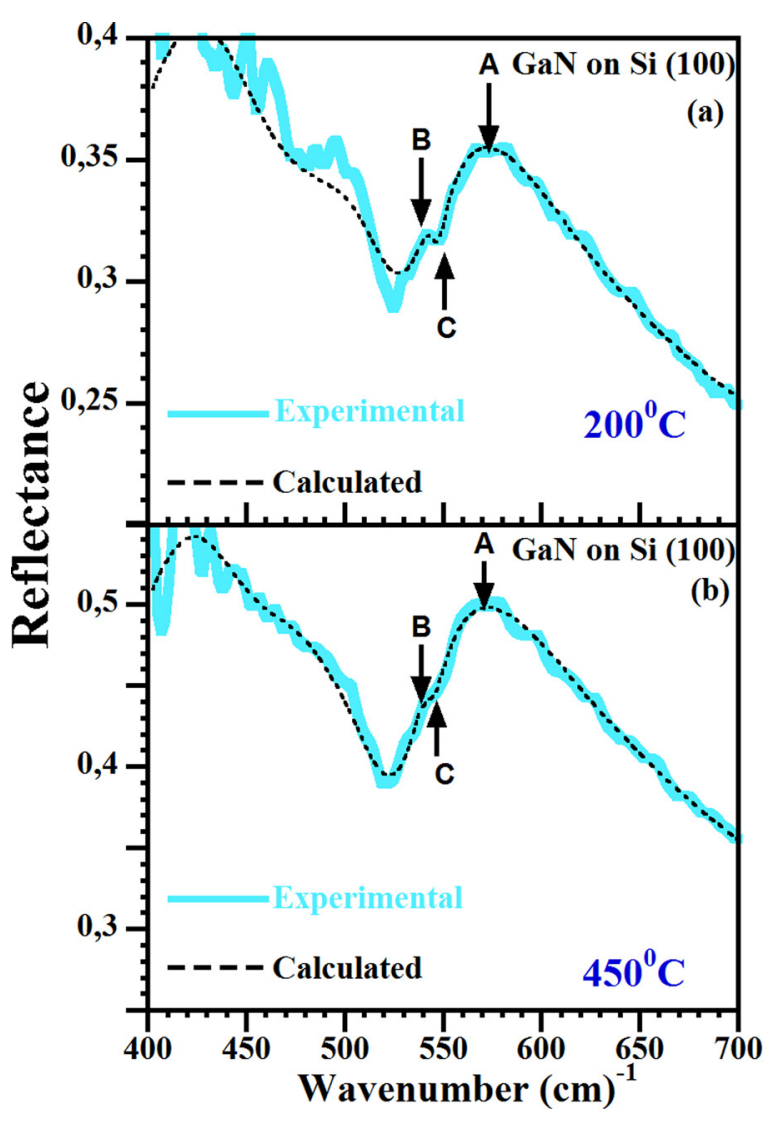

FIG. 6. (Color online) Experimental and calculated spectra IR reflectance spectra for $\mathrm{GaN}$ films deposited at substrate temperatures (a) $200^{\circ} \mathrm{C}$ and (b) $450{ }^{\circ} \mathrm{C}$. 
$\mathrm{Si}^{21}$ The main peak $\mathrm{A}$ at $\sim 570 \mathrm{~cm}^{-1}$ is due to the reflection from the $E_{1}(\mathrm{TO})$ phonon mode of wurtzite GaN. The $E_{1}(\mathrm{TO})$ frequencies obtained from the theoretical calculations are 555 and $553 \mathrm{~cm}^{-1}$ for GaN films deposited at 200 and $450{ }^{\circ} \mathrm{C}$, respectively. These assignments are close to the reported value for $E_{1}(\mathrm{TO})$, which is at $557 \mathrm{~cm}^{-1}$ in the literature. ${ }^{21}$ There is a peak located at point $\mathrm{B}$, which is seen clearly for GaN films deposited at $200^{\circ} \mathrm{C}$, and the peak is very weak for samples deposited at $450^{\circ} \mathrm{C}$ due to the rough surface morphology. ${ }^{21}$ The calculated wavenumber positions for peak B are 539 and $537 \mathrm{~cm}^{-1}$ for substrate temperatures 200 and $450^{\circ} \mathrm{C}$, respectively. The calculated value for peak $\mathrm{B}$ is quite close to the determined position for $\mathrm{A}_{1}(\mathrm{TO})$ phonon mode at $533 \mathrm{~cm}^{-1} \cdot{ }^{22}$ From the theoretical calculations, the wavenumber position obtained for dip $\mathrm{C}$ is 547 and $546 \mathrm{~cm}^{-1}$ for 200 and $450{ }^{\circ} \mathrm{C}$, respectively. The calculated wavenumber positions are not dependent on the substrate temperature, and the shift toward lower wave numbers is consistent with the compressive strain present in the $\mathrm{GaN}$ films grown in both self-limiting ALD and decomposition limited CVD growth regime.

\section{SUMMARY AND CONCLUSIONS}

We have studied hollow-cathode plasma-assisted atomic layer deposited crystalline GaN films in two main growth regimes: self-limited growth regime where growth rate per cycle stays constant with temperature (ALD region) and thermal decomposition-limited growth regime where growth rate increases with the substrate temperature (CVD region). As mentioned in GIXRD, AFM, spectroscopic ellipsometry, and optical transmission, crystallinity, microstructure, and surface roughnesses of these films grown with optimal growth parameters critically depend on the substrate temperatures. The increase in the substrate temperature leads to an increase in the growth rate, larger crystallite size, higher surface roughness, higher refractive index, and lower optical band gap value. It is found that there is a surface roughness layer including voids form with the increase in substrate temperature and correlates well with surface roughness of the films measured from the AFM images. The increase in optical band gap values calculated from spectroscopic ellipsometer and optical transmission data might be attributed to the film quality and preferred orientation of crystallites. ${ }^{17}$ Furthermore, the behavior of $E_{1}(\mathrm{TO})$ phonon modes were successfully described by a model using Lorentz oscillators for the dielectric function. The position of the $E_{1}(\mathrm{TO})$ phonon mode is independent of the substrate temperature, and the shift of $E_{1}(\mathrm{TO})$ phonon mode toward lower wavenumber was correlated and agrees well with the biaxial compressive strain present in both GaN films.

\section{ACKNOWLEDGMENTS}

M.A. acknowledges the financial support from TUBITAK Grant No. 114F002 and Marmara University BAPKO Project No. FEN-D-130515-0201. N.B. acknowledges the financial support from TUBITAK Grant Nos. 112M482 and 214M015. A.H. acknowledges Higher Education Commission of Pakistan (HEC) for Human Resource Development (HRD) fellowship.

${ }^{1}$ S. Nakamura and M. R. Krames, Proc. IEEE 101, 2211 (2013).

${ }^{2}$ M. Van Hove, S. Boulay, S. R. Bahl, S. Stoffels, X. Kang, D. Wellekens, K. Geens, A. Delabie, and S. Decoutere, IEEE Electron Device Lett. 33, 667 (2012).

${ }^{3}$ S. Nakamura, Jpn. J. Appl. Phys. 30, L1705 (1991).

${ }^{4}$ H. Amano, N. Sawaki, I. Akasaki, and N. Toyoda, Appl. Phys. Lett. 48, 353 (1986).

${ }^{5}$ M. E. Lin, B. Sverdlov, G. L. Zhou, and H. Morkoç, Appl. Phys. Lett. 62, 3479 (1993)

${ }^{6}$ P. Motamedi, N. Dalili, and K. Cadien, J. Mater. Chem. C 3, 7428 (2015).

${ }^{7}$ M. Alevli, R. Atalay, G. Durkaya, A. Weesekara, A. G. U. Perera, N. Dietz, R. Kirste, and A. Hoffmann, J. Vac. Sci. Technol. A 26, 1023 (2008).

${ }^{8}$ K. Shen, M. Jiang, H. Liu, H. Hsueh, Y. Kao, R. Horng, and D. Wuu, Opt. Express 21, 26468 (2013).

${ }^{9}$ M. Junaid, C. Hsiao, J. Palisaitis, J. Jensen, P. O. Persson, L. Hultman, and J. Birch, Appl. Phys. Lett. 98, 141915 (2011).

${ }^{10}$ C. Ozgit, I. Donmez, M. Alevli, and N. Biyikli, J. Vac. Sci. Technol. A 30, 01A124 (2012).

${ }^{11}$ H. Y. Shih, M. C. Lin, L. Y. Chen, and M. J. Chen, Nanotechnology 26, 014002 (2015).

${ }^{12}$ C. Ozgit-Akgun, E. Goldenberg, A. K. Okyay, and N. Biyikli, J. Mater. Chem. C 2, 2123 (2014).

${ }^{13}$ P. Motamedi and K. Cadien, RSC Adv. 5, 57865 (2015).

${ }^{14}$ V. Miikkulainen, M. Leskela, M. Ritala, and R. L. Puurunen, J. Appl. Phys. 113, 021301 (2013).

${ }^{15}$ K. M. A. Saron, M. R. Hashim, and M. A. Farrukh, Superlattice Microstruct. 64, 88 (2013).

${ }^{16}$ D. Li, M. Sumiya, S. Fuke, D. Yang, D. Que, Y. Suzuki, and Y. Fukuda, J. Appl. Phys. 90, 4219 (2001).

${ }^{17}$ A. Biswas, D. Bahttaccharyya, N. K. Sahoo, B. S. Yadav, S. S. Major, and R. S. Srinivasa, J. Appl. Phys. 103, 083541 (2008).

${ }^{18}$ H. Van Bui, F. B. Wiggers, A. Gupta, M. D. Nguyen, A. A. I. Aarnink, M. P. de Jong, and A. Y. Kovalgin, J. Vac. Sci. Technol. A 33, 01A111 (2015).

${ }^{19}$ A. B. Djurisic and E. H. Li, J. Appl. Phys. 85, 2848 (1999).

${ }^{20}$ A. Kasic, M. Schubert, S. Einfeldt, D. Hommel, and T. E. Tiwald, Phys. Rev. B 62, 7365 (2000).

${ }^{21}$ X. Zhang, Y. T. Hou, Z. C. Feng, and J. L. Chen, J. Appl. Phys. 89, 6165 (2001).

${ }^{22}$ Z. G. Hu, A. B. Weerasekara, N. Dietz, A. G. U. Perera, M. Strassburg, M. H. Kane, A. Asghar, and I. T. Ferguson, Phys. Rev. B 75, 205320 (2007). 